\title{
Association of FOXP3 gene polymorphisms with risk of preeclampsia in Lur population of Iran
}

\author{
Soheila Akbari $^{(\circledR)}$, Farhad Shahsavar ${ }^{\circledR}$, Babak Khodadadi ${ }^{\circledR}$, Seyyed Amir Yasin Ahmadi ${ }^{\circledR}$, Saber Abbaszadeh ${ }^{3,5}{ }^{\circledR}$, Seyyed \\ Ezatollah Rafiei Alavi ${ }^{* * \mathbb{C}}$ \\ 'Department of Obstetrics and Gynecology, Lorestan University of Medical Sciences, Khorramabad, Iran \\ ${ }^{2}$ Department of Immunology, Lorestan University of Medical Sciences, Khorramabad, Iran \\ ${ }^{3}$ Student Research Committee, Lorestan University of Medical Sciences, Khorramabad, Iran \\ ${ }^{4}$ Pediatric Growth and Development Research Center, Institute of Endocrinology and Metabolism, Iran University of Medical Sciences, \\ Tehran, Iran \\ ${ }^{5}$ Scientific Society of Evidence-based Knowledge, Research Office for the History of Persian Medicine, Lorestan University of Medical \\ Sciences, Khorramabad, Iran \\ ${ }^{6}$ Department of Pathology, Lorestan University of Medical Sciences, Khorramabad, Iran
}

*Correspondence to Seyyed Ezatollah Rafiei Alavi, Email: erafieialavi@gmail.com, rafieealavi@lums.ac.ir

Received 18 Sep. 2019 Accepted 15 Nov. 2019 Published online $14 \mathrm{Dec}$ 2019

Keywords: Preeclampsia, Regulatory T lymphocytes, FOXP3

\begin{abstract}
Introduction: Regulatory $\mathrm{T}$ lymphocytes have an effective role in induction of immune tolerance and angiogenesis during pregnancy. Differentiation and replication of these lymphocytes from other $\mathrm{T}$ cells is controlled by FOXP3 transcriptional factor. FOXP3 gene is polymorphic.

Objectives: This study was designed to investigate the role FOXP3 common polymorphisms in susceptibility to preeclampsia in Lur populations of Lorestan province of Iran.

Patients and Methods: This study was conducted on three polymorphisms rs2232365 A/G, rs3761548 C/A and rs5902434 del/ATT using polymerase chain reaction with sequence specific primers (PCR SSP). A total of 100 participants were subjected to be studied. Data analysis was done using Fisher's exact test and multivariate logistic regression.

Results: In rs2232365 polymorphism, AA genotype was a risk factor $(P=0.002)$ and AG genotype was a protecting factor $(P<0.001)$. In polymorphism of rs3761548, CA genotype was a risk factor $(P=0.036)$ and AA genotype was a protecting factor $(P=0.015)$. In polymorphism rs5902434, del/del genotype was a risk factor $(P=0.013)$ and del/ATT genotype was a protecting factor $(P<0.001)$. After adjusting the effects of genotypes, CA genotype of rs3761584 polymorphism was considered as an attributable risk factor $(P=0.040$; odds ratio [OR] $=4.19)$

Conclusion: The present population showed a unique association for the role of FOXP3 polymorphism in susceptibility to preeclampsia in comparison to previous studies.
\end{abstract}

\section{Introduction}

Preeclampsia is a pregnancy specific syndrome seen in about $5 \%$ of all pregnancies. Preeclampsia is an example of pregnancy hypertensive disorders which leads to maternal and fetal mortality and morbidity. Clinically, preeclampsia is called as a new onset hypertension after gestational age of 20 weeks along with a sign of organ damage which was previously defined as proteinuria. If there was no proteinuria or evidence of organ damage it is called gestational hypertension. In addition, chronic hypertension can be superimposed by preeclampsia (1). However, there are some unusual manifestations such as earlier hypertension or lack of hypertension in rare cases (2). The etiology of preeclampsia is not clear; however its pathophysiology has an immunological basis (3).

\begin{abstract}
Key point
Polymorphism of FOXP3 gene affects the risk of preeclampsia. This association is ethnicity dependent which may be due to haplotype effects. Therefore, from the viewpoint of medical anthropology these polymorphisms should be investigated in other diseases and populations to find immunological signature of ethnicities.
\end{abstract}

Accordingly, pregnancy is a semi-allograft transplantation. In such conditions, we need immune tolerance and induction of angiogenesis for a normal pregnancy. These functions are triggered by natural killer cells and regulatory $\mathrm{T}$ lymphocytes $(4,5)$. It has been observed that reduction in population of regulatory $\mathrm{T}$ cells increases the risk of pregnancy complications (6). Regulatory $\mathrm{T}$ cells are originated from Thelper- $0\left(\mathrm{CD}^{+}\right)$. T

\footnotetext{
Copyright (C) 2020 The Author(s); Published by Nickan Research Institute. This is an open-access article distributed under the terms of the Creative Commons Attribution License (http://creativecommons.org/licenses/by/4.0), which permits unrestricted use, distribution, and reproduction in any medium, provided the original work is properly cited.
} 
helper-0 is mainly differentiated to T helper-1, T helper-2, $\mathrm{T}$ helper-17 and regulatory $\mathrm{T}$ cells. Differentiation of regulatory $\mathrm{T}$ cells is initiated by transforming growth factor beta (TGF- $\beta$ ). TGF- $\beta$ results in the intracellular reactions which result in expression of a transcriptional factor named forkhead box protein 3 (FOXP3) from FOXP3 gene (7). Like many other genes, FOXP3 is polymorphic. This gene is located on chromosome X (8). Previously, induction of FOXP3 was proposed for treatment of infertility (9). Different hormones and vitamin D3 can induce the expression of FOXP3 $(10,11)$, and therefore, vitamin D deficiency increases risk of pregnancy complications (12).

FOXP3 gene has some common and uncommon polymorphisms. Previously, Song et al considered the three common polymorphisms of rs2232365 -924 A/G, rs3761548 -3297 C/A and rs5902434 -6054 del/ATT with risk of vitiligo (as an immune system related disease) in a Chinese population. They used logistic regression model and found that all of the above loci might influent the risk depending on the number of risk alleles from 0 to 6 (13). Therefore, these loci may be associated with risk of other diseases of immune system like preeclampsia.

\section{Objectives}

Single nucleotide polymorphisms (SNPs) of functional genes affect the susceptibility to different diseases. According to the critical role of FOXP3 in the function of regulatory $\mathrm{T}$ cells, the present study was aimed to investigate the association of FOXP3 SNPs with risk of preeclampsia in Lur population of Iran.

\section{Patients and Methods}

Study design

A genetic association study was performed with a casecontrol design. Genetic epidemiological approaches were used for interpretation of the data.

\section{Study population}

The sample size was calculated as 100 participants (50 per group) according to sample size calculation formula for comparison of proportions. During laboratory investigation, 20 samples were excluded because of lack of good quality DNA. The case groups were women with current or previous history of preeclampsia according to American Community of Obstetrics and Gynecology (ACOG) criteria including blood pressure $>140 / 90 \mathrm{~mm}$ $\mathrm{Hg}$ with onset after 20 weeks of gestational age, and having proteinuria (1+ in dipstick or $300 \mathrm{mg} / 24 \mathrm{~h}$ ) or evidence of organ damage instead. The severity of preeclampsia was not regarded. The exclusion criteria were having autoimmune disease or any history of pregnancy complication other than preeclampsia. The control group included women with history of at least two normal pregnancies without history of pregnancy complications. The samples were taken in Asali hospital, Khorramabad, Iran during 2018 using convenient sampling.

\section{Bioinformatics}

Among the common polymorphisms of FOXP3, three upstream SNPs including rs2232365 -924 A/G, rs3761548 -3297 C/A and rs5902434 -6054 del/ATT were selected. The details of these SNPs are available at www.snpedia. com. Sequence specific primers were used according to previous literature (Table 1) (13-15). The alleles left sided of the slashes were considered as wild type alleles.

\section{Laboratory investigation}

From each participant, $2 \mathrm{~mL}$ of peripheral blood was taken in EDTA containing tubes. The samples were kept at $-20^{\circ} \mathrm{C}$ till DNA extraction. DNA was extracted by sedimentation method using DNA extraction kit (Maxcell, Iran). Nanodrop spectrophotometer was used for calculating the concentration of DNA (which was $100-300 \mathrm{ng} / \mu \mathrm{L}$ ). The extracted DNA was kept at $-20^{\circ} \mathrm{C}$ till the reactions.

Polymerase chain reaction with sequence specific primers (PCR SSP) was used. For primary setup, PCR was performed according to thermal cycling protocol of Song et al for these primers (13). The PCR products were studied on agarose gel electrophoresis (Figure 1). After primary setup, high resolution melting master mix was used for reactions using real-time PCR. The PCR products were run on agarose gel, and melting curves were also studied. Each investigated allele for each sample was recorded as positive or negative.

\section{Ethical issues}

The research followed the tenets of the Declaration of Helsinki. Informed consent was taken from each participant. The personal data of the participants were kept in the hospital and deleted from the researcher archive. The present study was approved in the ethics committee of Lorestan University of Medical Sciences with registration number IR.LUMS.REC.1396.369. This study was a dissertation (M.D. Thesis) by Soheila Akbari at this

Table 1. Sequence specific primers used in the study

\begin{tabular}{lll}
\hline SNP & Forward primers & Reverse primers \\
\hline \multirow{2}{*}{ rs2232365 } & CCCAGCTCAAGAGACCCCA & Product size \\
& CCAGCTCAAGAGACCCCG & GGCTAGTGAGGAGGCTATTGTAAC \\
rs3761548 & CTGGCTCTCTCCCCAACTGA & GCTATAACAGTCCTGGCAAGTG \\
& TGGCTCTCTCCCCAACTGC & ACAGAGCCCATCATCAGACTCTCTA \\
\multirow{2}{*}{ rs5902434 } & ACCTTTAAGTCTTCTGCCATTTATTCTATTATTT & ACAGAGCCCATCATCAGACTCTCTA \\
& CCTTTAAGTCTTCTGCCATTTATTCTATTATTA & TGATTATCAGCGCACACACTCAT \\
\hline
\end{tabular}




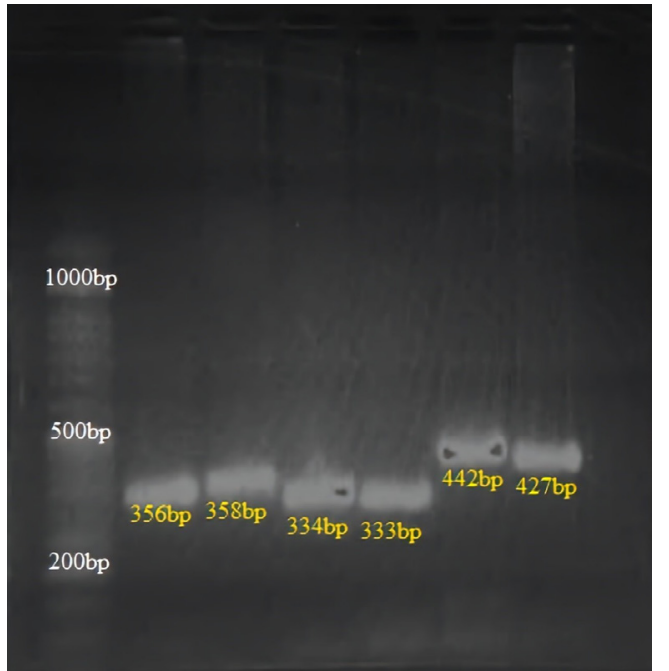

Figure 1. PCR products on agarose gel. The product sizes are according to table 1 .

university.

\section{Analysis of data}

Genotyping from the raw PCR data was performed using Excel 2013 software (Microsoft, US). Genotypes between the groups were compared using Fisher's exact test in 2 by 2 contingency tables. In order to control haplotype effect, multivariate logistic regression was used, in which all the 3 SNPs were the three independent variables. For each SNP, the most ineffective genotype (according to Fisher's exact test) was considered as the baseline genotype for logistic regression with odds ratio $(\mathrm{OR})=1$, and the second and third genotypes of each SNP were the risk and protective genotypes, respectively (according to Fisher's exact test). Inferential statistics were performed using Stata 14 software (StataCorp LLC, US).

\section{Results}

All the genotypes of all polymorphisms were detected in the present ethnicity. For polymorphism of rs2232365, frequency of the wild type allele (A) was more than 50\%. Frequency of homozygote genotypes was low in the control group. For polymorphism of rs3761548, frequency of the wild type allele (C) was less than $50 \%$. Frequency of the mutant genotype AA was more in the control group. For polymorphism of rs5902434, frequency of the wild type allele (del) was more than 50\%. Frequency of homozygote genotypes was low in the control group. Since the locus of FOXP3 was on chromosome X, it was not possible to calculate Hardy-Weinberg equilibrium with the conventional method. All the descriptive results mentioned above are without considering haplotype effect and confounding effect of the polymorphisms on each other (Table 2).

Frequency of genotypes was compared between groups. For polymorphism of rs2232365, wild homozygote genotype AA was a risk factor $(P=0.002)$ and heterozygote genotype AG was a protecting factor $(P<0.001)$. No significant result was observed for alleles. For polymorphism of rs3761548, mutant genotype AA was a protecting factor $(P=0.015)$ and genotype CA was a risk factor $(P=0.036)$. The mutant type allele $\mathrm{A}$ was a protecting factor $(P=0.048)$. For polymorphism rs5902434, wild homozygote genotype del/del was a risk factor $(P=0.013)$ and heterozygote genotype del/ATT was a protecting factor $(P<0.001)$. No significant result was observed for alleles (Table 3 ).

To find attributable genotypes and controlling haplotype effects, multivariate logistic regression was used. After running analysis, CA genotype from polymorphism of rs3761548 was found as the attributable genotype which was a risk factor for preeclampsia in this population $(P=0.040 ; \mathrm{OR}=4.10$ [in comparison to CC genotype] with 95\% confidence interval [CI]: 1.06-16.48). In addition, genotype AA from polymorphism rs2232365 showed a positive trend toward being a risk factor for preeclampsia $(P=0.056 ; \mathrm{OR}=8.34$ with $95 \% \mathrm{CI}: 0.96-73.63)$. No colinearity was observed between the polymorphisms after choosing baseline genotype for each. A significant

Table 2. Descriptive data of the distribution of genotypes and alleles

\begin{tabular}{|c|c|c|c|c|c|c|c|c|}
\hline \multirow{2}{*}{ SNP } & \multirow{2}{*}{ Genotype } & \multicolumn{3}{|c|}{ Group } & \multirow{2}{*}{ Allele } & \multicolumn{3}{|c|}{ Group } \\
\hline & & Control & Patient & Total & & Control & Patient & Total \\
\hline \multirow{4}{*}{ rs 2232365} & AA & $1(3.33)$ & $16(32.0)$ & $17(21.2)$ & A & $29(48.3)$ & $56(56.0)$ & 85 (53.1) \\
\hline & AG & $27(90.0)$ & $24(48.0)$ & $51(63.7)$ & G & $31(51.6)$ & $44(44.0)$ & 75 (46.8) \\
\hline & GG & $2(6.66)$ & $10(20.0)$ & $12(15.0)$ & Total & $60(100)$ & $100(100)$ & $160(100)$ \\
\hline & Total & $30(100)$ & $50(100)$ & $80(100)$ & & & & \\
\hline \multirow{4}{*}{ rs3761548 } & $\mathrm{AA}$ & $16(53.3)$ & $12(24.0)$ & $28(35.0)$ & A & $40(66.6)$ & $50(50.0)$ & $90(56.2)$ \\
\hline & $\mathrm{CA}$ & $8(26.6)$ & $26(52.0)$ & $34(42.5)$ & $\mathrm{C}$ & $20(33.3)$ & $50(50.0)$ & $70(43.7)$ \\
\hline & $\mathrm{CC}$ & $6(20.0)$ & $12(24.0)$ & $18(22.5)$ & Total & $60(100)$ & $100(100)$ & $160(100)$ \\
\hline & Total & $30(100)$ & $50(100)$ & $80(100)$ & & & & \\
\hline \multirow{4}{*}{ rs5902434 } & ATT/ATT & $2(6.66)$ & $12(24.0)$ & $14(17.5)$ & ATT & $28(46.6)$ & $42(42.0)$ & $70(43.7)$ \\
\hline & del/ATT & $24(80.0)$ & $18(36.0)$ & $42(52.5)$ & del & $32(53.3)$ & $58(58.0)$ & $90(56.2)$ \\
\hline & $\mathrm{del} / \mathrm{del}$ & $4(13.3)$ & $20(40.0)$ & $24(30.0)$ & Total & $60(100)$ & $100(100)$ & $160(100)$ \\
\hline & Total & $30(100)$ & $50(100)$ & 80 (100) & & & & \\
\hline
\end{tabular}

SNP, single nucleotide polymorphism. 
Table 3. Association of genotypes and alleles with risk of preeclampsia investigated by Fisher exact test

\begin{tabular}{lccc}
\hline SNP & Genotype/allele & Effect direction & P value \\
\hline \multirow{2}{*}{ rs2232365 } & AA & Risk & $0.002^{* *}$ \\
& AG & Protective & $<0.001^{* * *}$ \\
& GG & NS & 0.194 \\
Gs3761548 & CC & NS & 0.413 \\
& CA & Risk & 0.786 \\
& AA & Protective & $0.036^{*}$ \\
& A & Protective & $0.015^{*}$ \\
rs5902434 & del/del & Risk & $0.018^{*}$ \\
& del/ATT & Protective & $0<0.001^{* * *}$ \\
& ATT/ATT & NS & 0.068 \\
& ATT & NS & 0.622 \\
\hline
\end{tabular}

SNP, single nucleotide polymorphism.

* Significant at $P<0.05$; ** Significant at $P<0.01$; ** Significant at $P<0.001$.

interaction was found among the protecting genotypes of the three polymorphisms $(P=0.008$; OR $=0.187$ with $95 \%$ CI 0.054-0.643) (Table 4).

\section{Discussion}

The present genetic association study was designed to show the role of FOXP3 polymorphisms with susceptibility to preeclampsia as well as the effect of our ethnicity on this association. The unique results of this study in comparison to the previous ones showed the effect of ethnicity which may be due to haplotype effect. Haplotype effects can influent effects of individual genotypes $(16,17)$.

From the descriptive point of view, in two polymorphisms of rs2232365 and rs5902434 wild type alleles were most prevalent. For these two polymorphisms, frequency of homozygote genotypes was low. It shows that distribution of the genotypes of FOXP3 polymorphisms is ethnicity dependent.

According to the analyses of 2 by 2 tables, all three polymorphisms showed significant associations in susceptibility to preeclampsia. Controversial results of 2 by 2 tables and multivariate logistic regression shows that the effects of the attributable genotypes may be covered by other genotypes due to ethnicity related haplotypes. Therefore, the results of 2 by 2 tables are more reliable for in clinics whereas multivariate logistic regression analysis is more reliable to find cause-effect relations.

As we mentioned, unique results were found in comparison to the previous studies. Chen et al investigated the role of rs3761548 and rs5902434 polymorphisms in Han population of China (18). They found no significant association for the first polymorphism whereas for the second polymorphism they found del/del genotype as a protecting factor. In another study, they investigated rs2232365 polymorphism sine no significant association was observed (19). Jahan et al in India investigated the role of rs3761548 polymorphism. They found CA and CC genotypes act as risk factor s(20). Norouzian et al in Bandar
Table 4. Multivariate logistic regression for the effect of polymorphisms

\begin{tabular}{lcccc}
\hline SNP & Genotype & Odds ratio & $\mathbf{9 5} \% \mathbf{C l}$ & $\boldsymbol{P}$ value \\
\hline \multirow{2}{*}{ rs2232365 } & GG & 1 & Reference & \\
& AA & 8.34 & $0.94-73.63$ & 0.056 \\
& AG\# & 0.86 & $0.19-3.75$ & 0.843 \\
rs3761548 & CC & 1 & Reference & \\
& CA & 4.19 & $1.06-16.48$ & $0.040^{*}$ \\
& AA\# & 1.32 & $0.36-4.78$ & 0.665 \\
rs5902434 & ATT/ATT & 1 & Reference & \\
& del/del & 2.05 & $0.44-9.46$ & 0.355 \\
& del/ATT\# & 0.38 & $0.06-2.23$ & 0.286 \\
\hline
\end{tabular}

SNP, single nucleotide polymorphism.

* Significant at $P<0.05$; \# Significant interaction at $P<0.05$.

Note: The reference genotypes are the genotypes without effect direction according to Table 3 .

Abbas city of Iran investigated the role of rs2232365 and rs3761548 polymorphisms, while no significant association was observed (21). Gholami et al in Tehran investigated the role of rs2232365 and rs3761548 polymorphisms. For the first polymorphism they found AG genotype as a risk factor and for the second polymorphism they found AA genotype as a protecting factor (22). Although our study had been performed in Iran, however Lur population of Iran showed unique results even in comparison to other Iranian populations.

\section{Conclusion}

The present study showed a unique association for the polymorphisms of FOXP3 with susceptibility to preeclampsia in our ethnicity. From the viewpoint of basic medical sciences, immunological basis of preeclampsia was approved for another time. The given data cannot be representative of the gene pool of total Iranian population without comparison to other ethnic groups within the same study by using the same methods.

Limitations of the study

Effects of ethnicity related haplotypes might musk the effects of individual genotypes. Using multivariate logistic regression helped us to solve this problem. However, more powerful studies are suggested in other populations.

\section{Acknowledgments}

This work is a research project of SA with the same title. This project was used as the MD thesis of BK with ethics registration number IR.LUMS.REC.1396.369.

Authors' contribution

SA; sample taking and clinical supervision. FS; design and conceptualization. BK; search, writing and sample taking. SAYA; primary draft and analysis. SA; laboratory study. SERA; laboratory supervision and approval. All authors read and sign the final manuscript.

Conflicts of interest

The authors declare no conflict of interest. 


\section{Ethical considerations}

Ethical issues (including plagiarism, data fabrication, double publication) have been completely observed by the authors.

\section{Funding/Support}

Lorestan University of Medical Sciences supported the study (grant number A-10-1869-1).

\section{References}

1. Phipps E, Prasanna D, Brima W, Jim B. Preeclampsia: updates in pathogenesis, definitions, and guidelines. Clin J Am Soc Nephrol. 2016;11(6):1102-13. doi: 10.2215/CJN.12081115

2. Sanogo S, Konan SD, Yao KH, Diopoh SP, Aka J, Niava R. Preeclampsia without hypertension occurring at 17 weeks of amenorrhea; a case report and review of literature. J Nephropharmacol. 2018;7(2):169-73. doi: 10.15171/ npj.2018.33

3. Laresgoiti-Servitje E. A leading role for the immune system in the pathophysiology of preeclampsia. J Leukocyte Biol. 2013;94(2):247-57. doi: 10.1189/jlb.1112603

4. Alijotas-Reig J, Llurba E, Gris JM. Potentiating maternal immune tolerance in pregnancy: a new challenging role for regulatory T cells. Placenta. 2014;35(4):241-8. doi: 10.1016/j. placenta.2014.02.004

5. Torry DS, Leavenworth J, Chang M, Maheshwari V, Groesch $\mathrm{K}$, Ball ER, et al. Angiogenesis in implantation. J Assist Reprod Genet. 2007;24(7):303-15. doi: 10.1007/s10815-007-9152-7

6. La Rocca C, Carbone F, Longobardi S, Matarese G. The immunology of pregnancy: regulatory $\mathrm{T}$ cells control maternal immune tolerance toward the fetus. Immunol Lett. 2014;162(1):41-8. doi: 10.1016/j.imlet.2014.06.013

7. Chapoval S, Dasgupta P, Dorsey NJ, Keegan AD. Regulation of the T helper cell type $2(\mathrm{Th} 2) / \mathrm{T}$ regulatory cell (Treg) balance by IL-4 and STAT6. J Leukocyte Biol. 2010;87(6):1011-8. doi: 10.1189/jlb.1209772

8. Oda JM, Hirata BK, Guembarovski RL, Watanabe MA. Genetic polymorphism in FOXP3 gene: imbalance in regulatory T-cell role and development of human diseases. Journal of genetics. 2013;92(1):163-71. doi: 10.1007/s12041-013-0213-7

9. Guerin LR, Prins JR, Robertson SA. Regulatory T-cells and immune tolerance in pregnancy: a new target for infertility treatment? Hum Reprod Update. 2009;15(5):517-35. doi: 10.1093/humupd/dmp004

10. Chary AV, Hemalatha R, Seshacharyulu M, Murali MV, Jayaprakash D, Kumar BD. Vitamin D deficiency in pregnant women impairs regulatory $\mathrm{T}$ cell function. J Steroid Biochem Molecul Biol. 2015;147:48-55. doi: 10.1016/j. jsbmb.2014.11.020

11. Marinho A, Carvalho C, Boleixa D, Bettencourt A, Leal B,
Guimarães J, et al. Vitamin D supplementation effects on FoxP3 expression in $\mathrm{T}$ cells and FoxP3+/IL-17A ratio and clinical course in systemic lupus erythematosus patients: a study in a Portuguese cohort. Immunol Res. 2017;65(1):197206. doi: 10.1007/s12026-016-8829-3

12. Bodnar LM, Catov JM, Simhan HN, Holick MF, Powers RW, Roberts JM. Maternal vitamin D deficiency increases the risk of preeclampsia. Journal Clin Endocrinol Metabol. 2007;92(9):3517-22. doi: 10.1210/jc.2007-0718

13. Song P, Wang XW, Li HX, Li K, Liu L, Wei C, et al. Association between FOXP 3 polymorphisms and vitiligo in a Han Chinese population. British J Dermatol. 2013;169(3):571-8. doi: 10.1111/bjd.12377

14. Gao L, Li K, Li F, Li H, Liu L, Wang L, et al. Polymorphisms in the FOXP3 gene in Han Chinese psoriasis patients. J Dermatol Sci. 2010;57(1):51-6. doi: 10.1016/j.jdermsci.2009.09.010

15. Gholami M, Esfandiary A, Vatanparast M, Mirfakhraie R, Hosseini MM, Ghafouri-Fard S. Genetic variants and expression study of FOXP3 gene in acute coronary syndrome in Iranian patients. Cell Biochem Funct. 2016;34(3):158-62. doi: 10.1002/cbf.3174

16. Li L, Petsch K, Shimizu R, Liu S, Xu WW, Ying K, et al. Mendelian and non-Mendelian regulation of gene expression in maize. PLoS Genet. 2013;9(1):e1003202-e. doi: 10.1371/ journal.pgen.1003202

17. Epstein MP, Satten GA. Inference on haplotype effects in casecontrol studies using unphased genotype data. Am J Hum Genet. 2003;73(6):1316-29. doi: 10.1086/380204

18. Chen X, Gan T, Liao Z, Chen S, Xiao J. Foxp3 (-/ ATT) polymorphism contributes to the susceptibility of preeclampsia. PloS One. 2013;8(4):e59696. doi: 10.1371/ journal.pone.0059696

19. Chen X, Xu W, Chen Y, Liao Z, Gan T, Wu A, et al. Placental Foxp3 expression in patients with preeclampsia and correlation of Foxp3 gene locus 924 (rs2232365) polymorphism with preeclampsia. J South Med Univ. 2015;35(1):77-82

20. Jahan P, Sreenivasagari R, Goudi D, Komaravalli P, Ishaq M. Role of Foxp3 gene in maternal susceptibility to preeclampsia-A study from South India. Scandinavian J Immunol. 2013;77(2):104-8. doi: 10.1111/j.1365-3083.2012.02760.x

21. Norouzian M, Rahimzadeh M, Rajaee M, Arabpour F, Naderi $\mathrm{N}$. FoxP3 gene promoter polymorphism affects susceptibility to preeclampsia. Hum Immunol. 2016;77(12):1232-8. doi: 10.1016/j.humimm.2016.09.001

22. Gholami M, Mirfakhraie R, Pirjani R, Taheripanah R, Bayat $\mathrm{S}$, Daryabari SA, et al. Association study of FOXP3 gene and the risk of 0020 pre-eclampsia. Clin Experiment Hyperten. 2018;40(7):613-6. doi: 10.1080/10641963.2017.1411500 